\title{
The Existence and Simulations of Periodic Solution of Predator-Prey Models with Beddington-DeAngelis Functional Response and Impulsive Perturbations
}

\author{
Kaihua Wang \\ School of Mathematics and Statistics \\ Hainan Normal University \\ Haikou, Hainan, 571158, P.R. China \\ e-mail: kaihuawang@qq.com \\ Yan Yan \\ School of Mathematics and Statistics \\ Hainan Normal University \\ Haikou, Hainan, 571158, P.R. China \\ e-mail:20689967@qq.com
}

\author{
Zhanji Gui (Corresponding author) \\ Department of Software Engineering \\ Hainan College of Software Technology \\ Qionghai, Hainan571400, P.R. China \\ e-mail: zhanjigui@sohu.com
}

\begin{abstract}
In this paper, a predator-prey system with Beddington-DeAngelis functional response is studied, where the $n$ predators prey on one prey. Using the continuation theorem of coincidence degree theory and analysis techniques, a criteria for the existence of periodic solutions of predator-prey models with the Beddington-DeAngelis functional response governed by impulsive differential equations is established. Further, some numerical simulations show that our models can occur in many forms of complexities including periodic oscillation and Gui chaotic strange attractor.
\end{abstract}

Keywordscomponent Periodic-Solution; Predator-Prey Model; Coincidence degree theory; Impulses; BeddingtonDeAngelis Functional Response.

\section{INTRODUCTION}

Recently, many authors have devoted their efforts to the predator-prey system with Beddington-DeAngelis functional response which was introduced by Beddington and DeAngelis et al. independently (see [1-2]). It is well known that the traditional predator-prey systems with prey-dependent functional response fail to model the interference among predators. To overcome this shortcoming, Arditi and Ginzburg [3] proposed the ratiodependent predator-prey model which incorporates mutual interference by predators.

Although much progress has been seen in the study of predator-prey models with the Beddington-DeAngelis functional response (eg. [4,5]), such models are not well studied yet in the sense that all the existing results are based on the assumption that the predator preys on one prey. This assumption is rarely the case in real life. Naturally, more realistic and interesting model should take into account the predator preying on more than one prey. Therefore, Z.J. Zeng and M. Fan [6] established a more reasonable model with multiple preys. However, the corresponding problems (eg. [7]) with periodic coefficients and impulsive perturbations are studied far less often. In this paper, we will consider the following system:

$$
\left\{\begin{array}{c}
\frac{d x}{d t}=x(t)[a(t)-b(t) x(t) \\
\left.\quad-\sum_{i=1}^{n} \frac{c_{i}(t) y_{i}(t)}{\alpha_{i}(t)+\beta_{i}(t) x(t)+\gamma_{i}(t) y_{i}(t)}\right] \\
\frac{d y_{i}}{d t}=y_{i}(t)\left[-d_{i}(t)\right. \\
\left.+\frac{f_{i}(t) x(t)}{\alpha_{i}(t)+\beta_{i}(t) x(t)+\gamma_{i}(t) y_{i}(t)}\right]
\end{array}\right\} t t_{k},
$$

where $x(t)$ and $y_{i}(t)(i=1,2, \ldots, n)$ represent prey and predator densities respectively. $a(t)$ stands for prey intrinsic growth rate; $d_{i}(t)$ stands for the death rate of the predator; $c_{i}(t)$ and $f_{i}(t)$ are the uptake and predation constants of the predator and prey; $\alpha_{i}(t), \beta_{i}(t), \gamma_{i}(t)$ are positive constants; $p_{k}, q_{k}^{i}$ are constant.

In system (1), we assume:

(H1) $c_{i}(t), a(t), b(t), d_{i}(t), \alpha_{i}(t), \beta_{i}(t), \gamma_{i}(t)$ and $f_{i}(t)$ are all positive periodic continuous functions with period $\omega>0$.

(H2) There exist a positive constant $M$ and constant $N_{i}$ which satisfy $0 \leq N_{i} \leq 1$ such that $p_{k} \leq M, q_{k}^{i} \leq N_{i}$, $k=1,2$. .

(H3) $I_{k}=1+p_{k}>0, \bar{I}_{i k}=1+q_{k}^{i}>0$ and there exists a positive integer $q$ such that $t_{k+q}=t_{k}+\omega, q_{k+q}^{i}=q_{k}^{i}$, $p_{k+q}=p_{k},(i=1,2, \ldots, n)$. 


\section{EXISTENCE OF POSITIVE PERIODIC SOLUTIONS}

To prove our results, we need the notion of the Mawhin's continuation theorem formulated in [8].

Lemma 1 Let $X$ and $Y$ be two Banach spaces. Consider an operator equation $L x=\lambda N x$ where $L$ : Dom $L \cap X \rightarrow Y$ is a Fredholm operator of index zero and $\lambda \in[0,1]$ is a parameter. Let $P$ and $Q$ denote two projectors such that $P: X \rightarrow \operatorname{Ker} L$ and $Q: Y \rightarrow Y / \operatorname{Im} L$. Assume that $N: \bar{\Omega} \rightarrow Y$ is $L$-compact on $\bar{\Omega}$, where $\Omega$ is open bounded in $X$. Furthermore, assume that

(a) for each $\lambda \in(0,1), x \in \partial \Omega \cap \operatorname{Dom} L, L x \neq \lambda N x$;

(b) for each $x \in \partial \Omega \cap \operatorname{Ker} L, Q N x \neq 0$;

(c) $\operatorname{deg}\{J Q N x, \Omega \cap \operatorname{Ker} L, 0\} \neq 0$, where $J: \operatorname{Im} Q \rightarrow$

$\operatorname{Ker} L$ and $\operatorname{deg}\{*\}$ represents the Brouwer degree.

Then the equation $L x=N x$ has at least one solution in $\bar{\Omega} \cap \operatorname{Dom} L$.

For the sake of convenience, we introduce the following notations:

$$
\begin{gathered}
\bar{u}=\frac{1}{\omega} \int_{0}^{\omega} u(t) d t, \quad g_{i}^{l}=\min _{t \in[0, \omega]} g_{i}(t) \\
g_{i}{ }^{u}=\max _{t \in[0, \omega]} g_{i}(t), \quad(i=1,2, \ldots, n)
\end{gathered}
$$

Lemma $2 x^{*}(t)$ is an $\omega$-periodic solution of (1) if and only if $\ln \left\{x^{*}(t)\right\}$ is an $\omega$-periodic solution of

$$
\left\{\begin{aligned}
\frac{d x}{d t}= & a(t)-b(t) \exp \{x(t)\} \\
& -\sum_{i=1}^{n} \frac{c_{i}(t) \exp \left\{y_{i}(t)\right\}}{\alpha_{i}(t)+\beta_{i}(t) \exp \{x(t)\}+\gamma_{i}(t) \exp \left\{y_{i}(t)\right\}} \\
& +\frac{f_{i}(t) \exp \{x(t)\}}{\alpha_{i}(t)+\beta_{i}(t) \exp \{x(t)\}+\gamma_{i}(t) \exp \left\{y_{i}(t)\right\}}
\end{aligned}\right\} t \neq t_{k},
$$

Now we are ready to state and prove the main results of the present paper.

Theorem 1 Assume (H1)-(H3) hold, furthermore, we assume:

$$
\begin{gathered}
\left(\text { H4) } \begin{array}{c}
\left(\bar{f}_{i} \omega-\beta_{i}^{u}\left(\bar{d}_{i} \omega-p \ln \left(1-N_{i}\right)\right) \exp \left\{M_{1}\right\}\right. \\
>\alpha_{i}^{u}\left(\bar{d}_{i} \omega-p \ln \left(1-N_{i}\right)\right)
\end{array}\right. \\
\text { (H5) } \bar{a}-\sum_{i=1}^{n} \overline{c_{i} / \gamma_{i}}>0
\end{gathered}
$$

$$
\text { where } \quad M_{1}=\ln \frac{1}{\bar{b} \exp \{2 \bar{a} \omega\}(1+M)^{3 p}}\left(\bar{a}-\sum_{i=1}^{n} \overline{c_{i} / \gamma_{i}}\right) \text {, }
$$$$
(i=1,2 \ldots, n) \text {. }
$$

Then system (1) has at least one $\omega$-periodic solution.
Proof. To complete the proof, we only need to search for an appropriate open bounded subset $\Omega \subset X$ verifying all the requirements in Lemma 1.

Note $u=\left(x(t), y_{1}(t), \ldots, y_{n}(t)\right)^{T}$, obviously $I_{k} \geq 0$, $\bar{I}_{i k} \leq 0$. Define $X=\left\{u \in P C\left(,{ }^{n+1}\right) \mid u(t+\omega)=u(t)\right\}$, $Y=X \times i_{i}{ }^{2 p}$, then it is standard to show that both $X$ and $Y$ are Banach space when they are endowed with the norms $\quad\|u\|_{c}=\sup _{t \in[0, \omega]}|x(t)| \quad$ and $\quad\left\|\left(u, c_{1}, \ldots, c_{p}\right)\right\|=$ $\left(\|u\|_{p}^{2}+\left|c_{1}\right|^{2}+\ldots+\left|c_{p}\right|^{2}\right)^{1 / 2}$.

Let $\operatorname{Dom} L=X=\left\{u \in C^{1}\left[0, \omega, t_{1}, \ldots, t_{p}\right] \mid u(0)=u(\omega)\right\}$, $L: \operatorname{Dom} L \rightarrow Y, L x=\left(u^{\prime}, \Delta u\left(t_{1}\right), \ldots, \Delta u\left(t_{p}\right)\right)$,

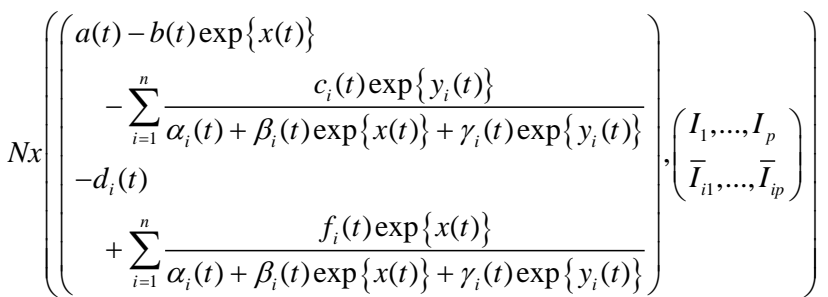

It is easy to prove that $L$ is a Fredholm mapping of index zero.

Consider the operator equation

$$
L x=\lambda N x \quad \lambda \in(0,1) \text {. }
$$

Integrating (4) over the interval $[0, \omega]$, we obtain

$$
\begin{gathered}
\bar{a} \omega=-\sum_{k=1}^{p} I_{k}+\int_{0}^{\omega} b(t) \exp \{x(t)\} d t \\
+\int_{0}^{\omega} \sum_{i=1}^{n} \frac{c_{i}(t) \exp \left\{y_{i}(t)\right\}}{\alpha_{i}(t)+\beta_{i}(t) \exp \{x(t)\}+\gamma_{i}(t) \exp \left\{y_{i}(t)\right\}} d t, \\
\bar{d}_{i} \omega=\sum_{k=1}^{p} \bar{I}_{i k} \\
\quad+\int_{0}^{\omega} \frac{f_{i}(t) \exp \{x(t)\}}{\alpha_{i}(t)+\beta_{i}(t) \exp \{x(t)\}+\gamma_{i}(t) \exp \left\{y_{i}(t)\right\}} d t, \\
\quad i=1,2, \ldots, n . \\
\int_{0}^{\omega}\left|x^{\prime}(t)\right| d t \leq 2 \bar{a} \omega+2 p \ln (1+M), \\
\int_{0}^{\omega}\left|y_{i}^{\prime}(t)\right| d t \leq \int_{0}^{\omega}\left|d_{i}(t)\right| d t \\
\quad+\int_{0}^{\omega}\left|\sum_{i=1}^{n} \frac{f_{i}(t) \exp \{x(t)\}}{\alpha_{i}(t)+\beta_{i}(t) \exp \{x(t)\}+\gamma_{i}(t) \exp \left\{y_{i}(t)\right\}}\right| d t \\
\quad+\sum_{k=1}^{p} \bar{I}_{i k} \\
\leq 2 \bar{d}_{i} \omega .
\end{gathered}
$$

Since $x(t) \in P C([0, \omega], \mathrm{i}), \quad y_{i}(t) \in P C([0, \omega], \mathrm{i})$, there exist $\xi, \eta, \xi_{i}, \eta_{i} \in[0, \omega] \cup\left[t_{1}^{+}, t_{2}^{+}, \ldots, t_{q}^{+}\right]$, such that $x(\xi)=\inf _{t \in[0, \omega]} x(t), \quad x(\eta)=\sup _{t \in[0, \omega]} x(t), \quad y_{i}\left(\xi_{i}\right)=\inf _{t \in[0, \omega]} y_{i}(t) \quad$, $y_{i}\left(\eta_{i}\right)=\sup _{t \in[0, \omega]} y_{i}(t), i=1,2, \ldots n$

From (5), we can see 


$$
\begin{aligned}
\bar{a} \omega & \leq \int_{0}^{\omega} b(t) \exp \{x(\eta)\} d t+ \\
& +\int_{0}^{\omega} \sum_{i=1}^{n} \frac{c_{i}(t) \exp \left\{y_{i}(t)\right\}}{\alpha_{i}(t)+\beta_{i}(t) \exp \{x(t)\}+\gamma_{i}(t) \exp \left\{y_{i}(t)\right\}} d t \\
& \leq \int_{0}^{\omega} b(t) \exp \{x(\eta)\} d t+\int_{0}^{\omega} \sum_{i=1}^{n} \frac{c_{i}(t) \exp \left\{y_{i}(t)\right\}}{\gamma_{i}(t) \exp \left\{y_{i}(t)\right\}} d t \\
& \leq \bar{b} \omega \exp \{x(\eta)\}+\sum_{i=1}^{n} \overline{c_{i} / \gamma_{i}} \omega .
\end{aligned}
$$

It follows that

$$
x(\eta) \geq \ln \frac{1}{\bar{b}}\left(\bar{a}-\sum_{i=1}^{n} \overline{c_{i} / \gamma_{i}}\right):=l_{1}
$$

$\int_{0}^{\omega} b(t) \exp \{x(\xi)\} \leq \int_{0}^{\omega} b(t) \exp \{x(t)\} \leq \bar{a} \omega+\sum_{k=1}^{p} I_{k}$.

$$
\bar{b} \omega \exp \{x(\xi)\} \leq \bar{a} \omega+p \ln (1+M) .
$$

It follows that

$$
x(\xi) \leq \ln \left[\frac{\bar{a} \omega+p \ln (1+M)}{\bar{b} \omega}\right]:=L_{1} .
$$

Then for $\forall t \in[0, \omega]$, we have

$$
\begin{gathered}
x(t) \geq x(\eta)-\sum_{k=1}^{p} I_{k}-\int_{0}^{\omega}\left|x^{\prime}(t)\right| d t \\
\geq l_{1}-2 \bar{a} \omega-3 p \ln (1+M) \geq M_{1}, \\
x(t) \leq x(\xi)+\sum_{k=1}^{p} I_{k}+\int_{0}^{\omega}\left|x^{\prime}(t)\right| d t \\
\leq \ln \left[\frac{(\bar{a} \omega+p \ln (1+M)) \exp \{2 \bar{a} \omega\}(1+M)^{3 p}}{\bar{b} \omega}\right]:=M_{2}
\end{gathered}
$$

then we can derive

$$
|x(t)| \leq \max \left\{\left|M_{1}\right|,\left|M_{2}\right|\right\}:=N_{1}
$$

Similarly, according to (4), for $\forall t \in[0, \omega]$, we have

$$
\begin{gathered}
\bar{d}_{i} \omega \leq \int_{0}^{\omega} \frac{f_{i}(t) \exp \{x(t)\}}{\alpha_{i}(t)+\beta_{i}(t) \exp \{x(t)\}+\gamma_{i}(t) \exp \left\{y_{i}(t)\right\}} d t \\
\leq \int_{0}^{\omega} \frac{f_{i}(t) \exp \left\{M_{2}\right\}}{\gamma_{i}^{l} \exp \left\{y_{i}\left(\xi_{i}\right)\right\}} d t \leq \frac{\bar{f}_{i} \omega \exp \left\{M_{2}\right\}}{\gamma_{i}^{l} \exp \left\{y_{i}\left(\xi_{i}\right)\right\}} . \\
y_{i}\left(\xi_{i}\right) \leq \ln \left[\frac{\bar{f}_{i} \exp \left\{M_{2}\right\}}{\bar{d}_{i} \gamma_{i}^{l}}\right]:=C_{i 1} .
\end{gathered}
$$

So, for $\forall t \in[0, \omega]$, we have

From (6), we also have

$$
\begin{aligned}
y_{i}(t) & \leq y_{i}\left(\xi_{i}\right)-\sum_{k=1}^{p} \bar{I}_{i k}+\int_{0}^{\omega}\left|y_{i}^{\prime}(t)\right| d t \\
& \leq C_{i 2}-p \ln \left(1-N_{i}\right)+2 \bar{d}_{i} \omega:=M_{i 3} .
\end{aligned}
$$

$$
\begin{aligned}
\bar{d}_{i} \omega-\sum_{k=1}^{p} \bar{I}_{i k} & =\int_{0}^{\omega} \frac{f_{i}(t) \exp \{x(t)\}}{\alpha_{i}(t)+\beta_{i}(t) \exp \{x(t)\}+\gamma_{i}(t) \exp \left\{y_{i}(t)\right\}} d t \\
& \geq \frac{\bar{f}_{i} \omega \exp \left\{M_{1}\right\}}{\alpha_{i}^{u}+\beta_{i}^{u} \exp \left\{M_{1}\right\}+\gamma_{i}^{u} \exp \left\{y_{i}\left(\eta_{i}\right)\right\}},
\end{aligned}
$$

then we can derive

$$
y_{i}\left(\eta_{i}\right) \geq \ln \left[\frac{S}{\gamma_{i}^{u}\left(\bar{d}_{i} \omega-p \ln \left(1-N_{i}\right)\right)}\right]:=C_{i 2},
$$

where

$$
\begin{gathered}
S=\left(\bar{f}_{i} \omega-\beta_{i}^{u}\left(\bar{d}_{i} \omega-p \ln \left(1-N_{i}\right)\right) \exp \left\{M_{1}\right\}\right. \\
-\alpha_{i}^{u}\left(\bar{d}_{i} \omega-p \ln \left(1-N_{i}\right)\right) .
\end{gathered}
$$

Thus

$$
\begin{aligned}
y_{i}(t) & \geq y_{i}\left(\eta_{i}\right)+\sum_{k=1}^{p} \bar{I}_{i k}-\int_{0}^{\omega}\left|y_{i}^{\prime}(t)\right| d t \\
& \geq C_{i 2}+p \ln \left(1-N_{i}\right)-2 \bar{d}_{i} \omega:=M_{i 4} .
\end{aligned}
$$

Then we can derive

$$
\left|y_{i}(t)\right| \leq \max \left\{\left|M_{i 3}\right|,\left|M_{i 4}\right|\right\}:=N_{2}
$$

Obviously, there exists a constant $N_{3}>0$ such that $\max \{|\bar{x}|,|\bar{y}|, i=1,2, \ldots, n\}<N_{3}$. Take $r>N_{1}+N_{2}+$ $N_{3}, \Omega=\left\{x \in X:\|x\|_{c}<r\right\}$ then $N$ is $L$-compact on $\bar{\Omega}$. So, for $\forall u=\left(\bar{x}, \bar{y}_{1}, \bar{y}_{2}, \ldots, \bar{y}_{n}\right)^{T} \in \partial \Omega \cap \operatorname{Ker} L$, we have $Q N x \neq 0$. Let $J: \operatorname{Im} Q \rightarrow X,(d, 0, \ldots, 0) \rightarrow d$. Then for $\forall u \in \Omega \cap \operatorname{Ker} L$, in view of the assumptions in Mawhin's continuation theorem [8], one obtains $\operatorname{deg}\{J Q N x, \Omega \cap \operatorname{Ker} L, 0\} \neq 0$. By now we have proved that $\Omega$ satisfies all the requirements in Mawhin's continuation theorem. Hence, system (3) has at least one $\omega$-periodic solution. By Lemma 2, we derive that system (1) has at least one positive $\omega$-periodic solution. The proof is complete.

\section{AN ILLUSTRATIVE EXAMPLE}

In this section, we shall discuss an example to illustrate our main results. In (1), we take $n=2, t_{k}=k T$,

$$
\begin{gathered}
a(t)=3-\sin (t), \quad d_{1}(t)=1+0.5 \cos (t), \\
c_{1}(t)=1-0.3 \cos (t), \quad f_{1}(t)=5+2 \sin (t), \\
b(t)=1+0.2 \cos (t), \quad d_{2}(t)=1-0.1 \sin (t), \\
c_{2}(t)=0.7+0.3 \sin (t), \quad f_{2}(t)=4+3 \cos (t), \\
\alpha_{1}(t)=3-0.3 \sin (t), \quad \beta_{1}(t)=1+0.3 \cos (t), \\
\gamma_{1}(t)=0.8-0.5 \sin (t), \quad \alpha_{2}(t)=2+0.2 \sin (t), \\
\beta_{2}(t)=1+0.2 \sin (t), \quad \gamma_{2}(t)=1+0.5 \cos (t) .
\end{gathered}
$$

If $p_{k}=0.3, q_{k}^{1}=0.2, q_{k}^{2}=0.3$, all conditions of Theorem 2 are satisfied. If $T=\pi$, then system (1) under has a unique periodic solution (see Fig .1-Fig .4). Because of the influence of the period pulses, the influence of pulse is obvious.

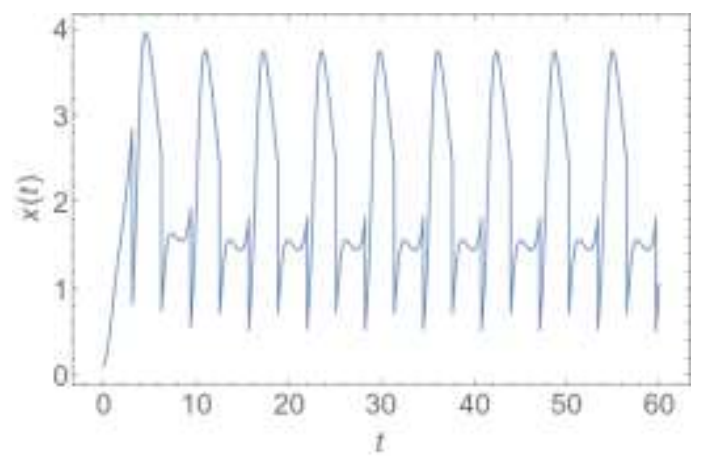

Figure 1. Time-series of $x(t)$ of system (1) with $T=\pi$. 


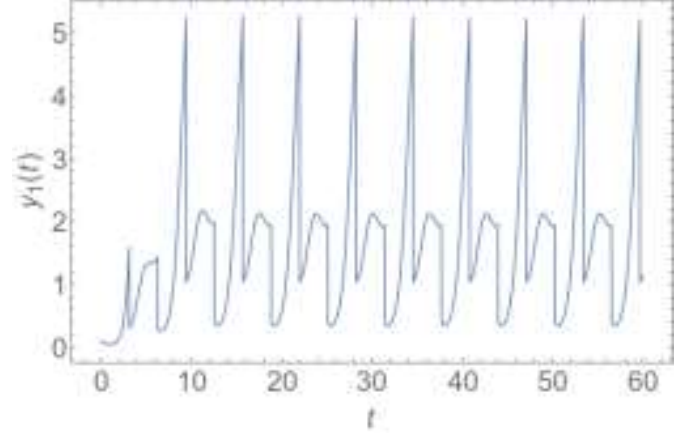

Figure 2. Time-series of $y_{1}(t)$ of system (1) with $T=\pi$.

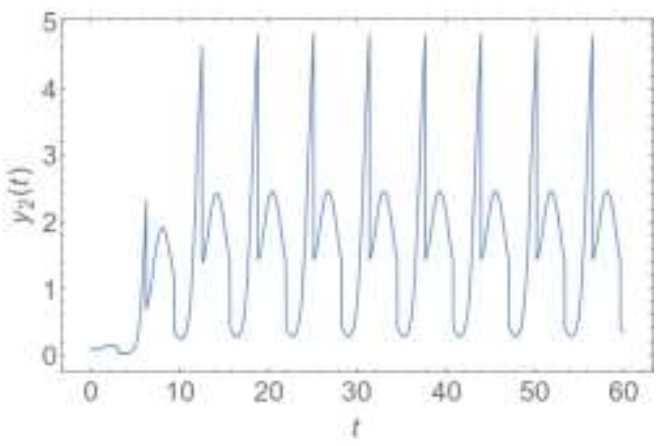

Figure 3. Time-series of $y_{2}(t)$ of system (1) with $T=\pi$.

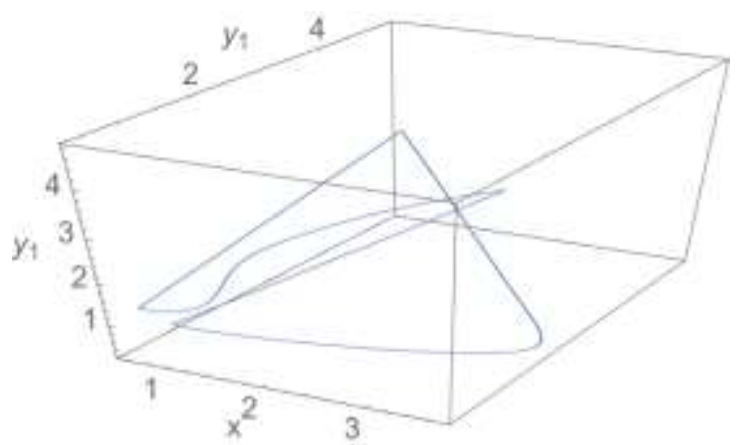

Figure 4. Phase portrait of periodic solution of system (1).

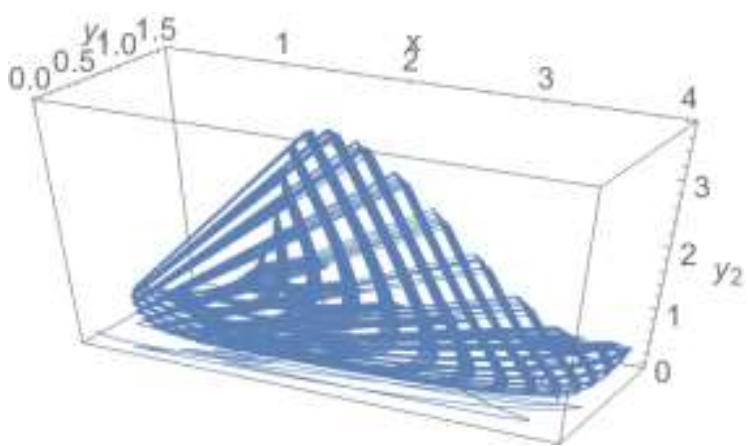

Figure 5. Phase portrait of Gui strange attractor of system (1).
If $T=2$, then (H3) is not satisfied. Periodic oscillation of system (1) will be destroyed by impulsive effect. Numeric results show that system (1) has Gui chaotic strange attractor [9-13], see Fig .5.

\section{ACKNOWLEDGMENT}

This work is supported jointly by the Natural Sciences Foundation of China under Grant No. 60963025, Natural Sciences Foundation of Hainan Province under Grant No.613166.

\section{REFERENCES}

[1] J.R. Beddington, "Mutual interference between parasites or predators and its effect on searching efficiency," J. Animal Ecol. vol. 44, Feb. 1975, pp. 331-340.

[2] D.L. DeAngelis, R.A. Goldstein, R.V. O'Neill, "A model for tropic interaction," Ecology, vol. 56, Feb. 1975, pp. 881-892.

[3] R. Arditi, L.R. Ginzburg, "Coupling in predator-prey dynamics: Ratio-dependence," J. Theoret. Biol. vol. 139, Aug. 1989, pp. 311 326, doi:10.1016/S0022-5193(89)80211-5.

[4] R. Cantrell and C. Cosner, "On the dynamics of predator-prey models with the Beddington-DeAngelis functional response." Journal of Mathematical Analysis and Applications, vol. 257, May 2001, pp. 206-222, doi:10.1006/jmaa.2000.7343.

[5] W.S. Yang, X.P. Li, "Global asymptotical stability for a diffusive predator-prey system with Beddington-DeAngelis functional response and nonlocal delay," Journal of Applied Mathematics and Computing, Feb. 2015, pp. 1-21, doi: 10.1007/s12190-015-0873-y.

[6] Z.J. Zeng, M. Fan, "Study on a non-autonomous predator-prey system with Beddington-DeAngelis functional response," Mathematical and Computer Modelling, vol. 48, Jul. 2008, pp. 1755-1764, doi:10.1016/j.mcm.2008.05.052.

[7] S. Sarwardi, M.R. Mandal, N.H. Gazi, "Dynamical behaviour of an ecological system with Beddington-DeAngelis functional response", Jan. 2015, eprint arXiv:1501.04847.

[8] R.E. Gaines, J.L. Mawhin, Coincidence degree and nonlinear deferential equations. Springer-Verlag, Berlin. 1977.

[9] J. Zhang and Z.J. Gui, "Existence and stability of periodic solutions of high-order Hopfield neural networks with impulses and delays," Journal of Computational and Applied Mathematics, vol. 224, Feb. 2009, pp. 602-613, doi:10.1016/j.cam.2008.05.042.

[10] J. Zhang and Z.J. Gui, "Periodic solutions of nonautonomous cellular neural networks with impulses and delays," Nonlinear Analysis: Real World Applications, vol. 10, Jun. 2009, pp. 18911903, doi:10.1016/j.nonrwa.2008.02.029

[11] C.B. Xing and Z.J. Gui, "Periodic Solution of Impulsive predatorprey System with Holling-Tanner Type Functional Response," In L. Chen, et al (eds.). Advances on Biomathematics. Liverpool: World Academic Union (World Academic Press). 2008, pp.58-62.

[12] J. Zhang and Z.J. Gui, "Periodic Solution of Predator-Prey Models with the Beddington-DeAngelis Functional Response Governed by Impulsive Differential Equations," In: L. Chen, et al (eds.) Advances on Biomathematics. Liverpool: World Academic Union (World Academic Press). 2008, pp265-268.

[13] K.H. Wang, Z.J. Gui, "Existence and Simulations of Periodic Solution of a three-species Lotka-Volterra Predator-prey System with Impulsive Perturbations", Proceedings of 2012 IEEE International Conference on Computer Science and Automation Engineering, 2012, 10.1109/CSAE.2012.6272934 